\title{
Solution of the First Boundary-Value Problem for a System of Autonomous Second-Order Linear Partial Differential Equations of Parabolic Type with a Single Delay
}

\author{
Josef Diblik, ${ }^{1,2}$ Denis Khusainov, ${ }^{3}$ \\ Oleksandra Kukharenko, ${ }^{3}$ and Zdeněk Svoboda ${ }^{2}$ \\ ${ }^{1}$ Department of Mathematics and Descriptive Geometry, Faculty of Civil Engineering, Brno University of \\ Technology, 60200 Brno, Czech Republic \\ ${ }^{2}$ Department of Mathematics, Faculty of Electrical Engineering and Communication, Brno University of \\ Technology, 61600 Brno, Czech Republic \\ ${ }^{3}$ Department of Cybernetics, Taras Shevchenko National University of Kyiv, Kyiv 01601, Ukraine
}

Correspondence should be addressed to Josef Diblík, diblik@feec.vutbr.cz

Received 29 March 2012; Accepted 9 May 2012

Academic Editor: Miroslava Růžičková

Copyright (C) 2012 Josef Diblík et al. This is an open access article distributed under the Creative Commons Attribution License, which permits unrestricted use, distribution, and reproduction in any medium, provided the original work is properly cited.

The first boundary-value problem for an autonomous second-order system of linear partial differential equations of parabolic type with a single delay is considered. Assuming that a decomposition of the given system into a system of independent scalar second-order linear partial differential equations of parabolic type with a single delay is possible, an analytical solution to the problem is given in the form of formal series and the character of their convergence is discussed. A delayed exponential function is used in order to analytically solve auxiliary initial problems (arising when Fourier method is applied) for ordinary linear differential equations of the first order with a single delay.

\section{Introduction}

In this paper, we deal with an autonomous second-order system of linear partial differential equations of the parabolic type with a single delay

$$
\begin{aligned}
& \frac{\partial u(x, t)}{\partial t}=a_{11} \frac{\partial^{2} u(x, t-\tau)}{\partial x^{2}}+a_{12} \frac{\partial^{2} v(x, t-\tau)}{\partial x^{2}}+b_{11} \frac{\partial^{2} u(x, t)}{\partial x^{2}}+b_{12} \frac{\partial^{2} v(x, t)}{\partial x^{2}} \\
& \frac{\partial u(x, t)}{\partial t}=a_{21} \frac{\partial^{2} u(x, t-\tau)}{\partial x^{2}}+a_{22} \frac{\partial^{2} v(x, t-\tau)}{\partial x^{2}}+b_{21} \frac{\partial^{2} u(x, t)}{\partial x^{2}}+b_{22} \frac{\partial^{2} v(x, t)}{\partial x^{2}}
\end{aligned}
$$


where the matrices of coefficients

$$
A=\left(\begin{array}{ll}
a_{11} & a_{12} \\
a_{21} & a_{22}
\end{array}\right), \quad B=\left(\begin{array}{ll}
b_{11} & b_{12} \\
b_{21} & b_{22}
\end{array}\right)
$$

are constant and $\tau>0, \tau=$ const.

Usually, when systems of differential equations are investigated, the main attention is paid to systems of ordinary differential equations or systems of partial differential equations [1-5]. The analysis of systems of partial differential equations with delay is rather neglected. This investigation is extremely rare.

The first boundary-value problem for (1.1) is solved for $A$ having real eigenvalues $\lambda_{1}$, $\lambda_{2}$, and $B$ having real eigenvalues $\sigma_{1}>0, \sigma_{2}>0$. Throughout the paper, we assume that there exists a real constant regular matrix

$$
S=\left(\begin{array}{ll}
s_{11} & s_{12} \\
s_{21} & s_{22}
\end{array}\right)
$$

simultaneously reducing both matrices $A$ and $B$ into diagonal forms

$$
\Lambda=\left(\begin{array}{cc}
\lambda_{1} & 0 \\
0 & \lambda_{2}
\end{array}\right), \quad \Sigma=\left(\begin{array}{cc}
\sigma_{1} & 0 \\
0 & \sigma_{2}
\end{array}\right)
$$

that is,

$$
S^{-1} A S=\Lambda, \quad S^{-1} B S=\Sigma,
$$

where

$$
S^{-1}=\frac{1}{\Delta}\left(\begin{array}{cc}
s_{22} & -s_{12} \\
-s_{21} & s_{11}
\end{array}\right), \quad \Delta=s_{11} s_{22}-s_{12} s_{22}
$$

For some classes of matrices, suitable transformations are known. Let us mention one of such results [6, Theorem $11^{\prime}$, page 291]. First, we recall that a complex square matrix $\mathcal{A}$ is a normal matrix if $\mathscr{A}^{*} \mathscr{A}=A_{A} \mathscr{A}^{*}$ where $\mathscr{A}^{*}$ is the conjugate transpose of $\mathcal{A}$. If $\mathscr{A}$ is a real matrix, then $\mathcal{A}^{*}=\mathcal{A}^{T}$, that is, the real matrix is normal if $\mathscr{A}^{T} \mathscr{A}=\mathscr{A} \mathcal{A}^{T}$. A square matrix $\mathfrak{U}$ is called unitary if $\mathfrak{U} \mathfrak{U}^{*}=E$ where $E$ is the identity matrix.

Theorem 1.1. If a finite or infinite set of pairwise commuting normal matrices is given, then all these matrices can be carried by one and the same unitary transformation into a diagonal form.

Let $l$ be a positive constant, and let

$$
\begin{gathered}
\mu_{i}:[-\tau, \infty) \longrightarrow \mathbb{R}, \quad i=1,2 \\
\theta_{i}:[-\tau, \infty) \longrightarrow \mathbb{R}, \quad i=1,2, \\
\varphi:[0, l] \times[-\tau, 0] \longrightarrow \mathbb{R}, \\
\psi:[0, l] \times[-\tau, 0] \longrightarrow \mathbb{R}
\end{gathered}
$$


be continuously differentiable functions such that

$$
\begin{array}{ll}
\mu_{1}(t)=\varphi(0, t), & t \in[-\tau, 0], \\
\mu_{2}(t)=\varphi(l, t), & t \in[-\tau, 0], \\
\theta_{1}(t)=\psi(0, t), & t \in[-\tau, 0], \\
\theta_{2}(t)=\psi(l, t), & t \in[-\tau, 0] .
\end{array}
$$

Together with system (1.1), we consider the first boundary-value problem, that is, the boundary conditions

$$
\begin{array}{ll}
u(0, t)=\mu_{1}(t), & t \in[-\tau, \infty), \\
u(l, t)=\mu_{2}(t), & t \in[-\tau, \infty), \\
v(0, t)=\theta_{1}(t), & t \in[-\tau, \infty), \\
v(l, t)=\theta_{2}(t), & t \in[-\tau, \infty)
\end{array}
$$

and the initial conditions

$$
\begin{aligned}
& u(x, t)=\varphi(x, t), \quad(x, t) \in[0, l] \times[-\tau, 0], \\
& v(x, t)=\psi(x, t), \quad(x, t) \in[0, l] \times[-\tau, 0] .
\end{aligned}
$$

A solution to the first boundary-value problem (1.1), (1.9)-(1.14) is defined as a pair of functions

$$
u, v:[0, l] \times[-\tau, \infty) \longrightarrow \mathbb{R},
$$

continuously differentiable with respect to variable $t$ if $(x, t) \in[0, l] \times[0, \infty)$, twice continuously differentiable with respect to $x$ if $(x, t) \in[0, l] \times[0, \infty)$, satisfying the system (1.1) for $(x, t) \in[0, l] \times[0, \infty)$, the boundary conditions (1.9)-(1.12), and the initial conditions (1.13), (1.14). If necessary, we restrict the above definition of the solution to $(x, t) \in[0, l] \times$ $[-\tau, k \tau]$ where $k$ is a positive integer.

The purpose of the paper is to describe a method of constructing a solution of the above boundary-initial problem. Assuming that a decomposition of system (1.1) into a system of independent scalar second-order linear partial differential equations of parabolic type with a single delay is possible, an analytical solution to the problem (1.9)-(1.14) is given in the form of formal series in part 3 . Their uniform convergence as well as uniform convergence of the partial derivatives of a formal solution is discussed in part 4 . A delayed exponential function (defined in part 2 together with the description of its main properties) is used in order to analytically solve auxiliary initial problems (arising when Fourier method is applied) for ordinary linear differential equations of the first-order with a single delay. 
To demonstrate this method, we will use systems of two equations only, although it can simply be extended to systems of $n$ equations.

\section{Preliminaries-Representation of Solutions of Linear Differential Equations with a Single Delay}

A solution of the systems (1.1) satisfying all boundary and initial conditions (1.9)-(1.14) will be constructed by the classical method of separation of variables (Fourier method). Nevertheless, due to delayed arguments, complications arise in solving analytically auxiliary initial Cauchy problems for first-order linear differential equations with a single delay. We overcome this circumstance by using a special function called a delayed exponential, which is a particular case of the delayed matrix exponential (as defined, e.g., in [7-10]). Here we give a definition of the delayed exponential, its basic properties needed, and a solution of the initial problem for first-order homogeneous and nonhomogeneous linear differential equations with a single delay.

Definition 2.1. Let $b \in \mathbb{R}$. The delayed exponential function $\exp _{\tau}\{b, t\}: \mathbb{R} \rightarrow \mathbb{R}$ is a function continuous on $\mathbb{R} \backslash\{-\tau\}$ defined as

$$
\exp _{\tau}\{b, t\}= \begin{cases}0 & \text { if }-\infty<t<-\tau, \\ 1 & \text { if }-\tau \leq t<0, \\ 1+b \frac{t}{1 !} & \text { if } 0 \leq t<\tau, \\ \cdots & \\ 1+b \frac{t}{1 !}+b^{2} \frac{(t-\tau)^{2}}{2 !}+\cdots+b^{k} \frac{(t-(k-1) \tau)^{k}}{k !} & \text { if }(k-1) \tau \leq t<k \tau, \\ \cdots & \end{cases}
$$

where $k=0,1,2, \ldots$.

Lemma 2.2. For the differentiation of a delayed exponential function, the formula

$$
\frac{d}{d t} \exp _{\tau}\{b, t\}=b \exp _{\tau}\{b, t-\tau\}
$$

holds within every interval $(k-1) \tau \leq t<k \tau, k=0,1,2, \ldots$

Proof. Within the intervals $(k-1) \tau \leq t<k \tau, k=0,1,2, \ldots$, the delayed exponential function is expressed as

$$
\exp _{\tau}\{b, t\}=1+b \frac{t}{1 !}+b^{2} \frac{(t-\tau)^{2}}{2 !}+b^{3} \frac{(t-2 \tau)^{3}}{3 !}+\cdots+b^{k} \frac{(t-(k-1) \tau)^{k}}{k !}
$$


Differentiating this expression, we obtain

$$
\begin{aligned}
\frac{d}{d t} \exp _{\tau}\{b, t\} & =b+b^{2} \frac{t-\tau}{1 !}+b^{3} \frac{(t-2 \tau)^{2}}{2 !}+\cdots+b^{k} \frac{(t-(k-1) \tau)^{k-1}}{(k-1) !} \\
& =b\left[1+b \frac{t-\tau}{1 !}+b^{2} \frac{(t-2 \tau)^{2}}{2 !}+\cdots+b^{k-1} \frac{[t-(k-1) \tau]^{k-1}}{(k-1) !}\right] \\
& =b \exp _{\tau}\{b, t-\tau\} .
\end{aligned}
$$

\subsection{First-Order Homogeneous Linear Differential Equations with a Single Delay}

Let us consider a linear homogeneous equation with a single delay

$$
\dot{x}(t)=b x(t-\tau),
$$

where $b \in \mathbb{R}$, together with the initial Cauchy condition

$$
x(t)=\beta(t), \quad t \in[-\tau, 0] .
$$

From (2.2), it immediately follows that the delayed exponential $\exp _{\tau}\{b, t\}$ is a solution of the initial Cauchy problems (2.5), (2.6) with $\beta(t) \equiv 1, t \in[-\tau, 0]$.

Theorem 2.3. Let $\beta:[-\tau, 0] \rightarrow \mathbb{R}$ be a continuously differentiable function. Then the unique solution of the initial Cauchy problems (2.5), (2.6) can be represented as

$$
x(t)=\exp _{\tau}\{b, t\} \beta(-\tau)+\int_{-\tau}^{0} \exp _{\tau}\{b, t-\tau-s\} \beta^{\prime}(s) d s,
$$

where $t \in[-\tau, \infty)$.

Proof. The representation (2.7) is a linear functional of the delayed exponential function $\exp _{\tau}\{b, t\}$ and $\exp _{\tau}\{b, t-\tau-s\}$. Because by Lemma 2.2 the delayed exponential function is the solution of (2.5), the functional on the right-hand side of (2.7) is a solution of the homogeneous equation (2.5) for arbitrary (differentiable) $\beta(t)$.

We will show that initial condition (2.6) is satisfied as well, that is, we will verify that, for $-\tau \leq t \leq 0$, the next identity is correct:

$$
\beta(t) \equiv \exp _{\tau}\{b, t\} \beta(-\tau)+\int_{-\tau}^{0} \exp _{\tau}\{b, t-\tau-s\} \beta^{\prime}(s) d s .
$$

We rewrite (2.7) as

$$
x(t)=\exp _{\tau}\{b, t\} \beta(-\tau)+\int_{-\tau}^{t} \exp _{\tau}\{b, t-\tau-s\} \beta^{\prime}(s) d s+\int_{t}^{0} \exp _{\tau}\{b, t-\tau-s\} \beta^{\prime}(s) d s .
$$


From Definition 2.1, it follows:

$$
\begin{gathered}
\exp _{\tau}\{b, t\} \equiv 1 \quad \text { if } \quad-\tau \leq t \leq 0 \\
\exp _{\tau}\{b, t-\tau-s\} \equiv 1 \quad \text { if }-\tau \leq s \leq t \\
\exp _{\tau}\{b, t-\tau-s\} \equiv 0 \quad \text { if } t<s<0
\end{gathered}
$$

Therefore,

$$
x(t)=\beta(-\tau)+\int_{-\tau}^{t} \beta^{\prime}(s) d s=\beta(-\tau)+\beta(t)-\beta(-\tau)=\beta(t) .
$$

Remark 2.4. Computing the integral in formula (2.7) by parts, we obtain for $t \geq \tau$ :

$$
x(t)=\exp _{\tau}\{b, t-\tau\} \beta(0)+b \int_{-\tau}^{0} \exp _{\tau}\{b, t-2 \tau-s\} \beta(s) d s .
$$

We remark that it is possible to prove this formula assuming only continuity of the function $\beta$, that is, continuous differentiability of $\beta$ is, in general, not necessary when we represent $x$ by formula (2.12).

Further we will consider the linear nonhomogeneous differential equation with a single delay

$$
\dot{x}(t)=a x(t)+b x(t-\tau),
$$

where $a, b \in \mathbb{R}$, together with initial Cauchy condition (2.6).

Theorem 2.5. Let the function $\beta$ in (2.6) be continuously differentiable. Then the unique solution of the initial Cauchy problems (2.13), (2.6) can be represented as

$$
x(t)=\exp _{\tau}\left\{b_{1}, t\right\} e^{a(t+\tau)} \beta(-\tau)+\int_{-\tau}^{0} \exp _{\tau}\left\{b_{1}, t-\tau-s\right\} e^{a(t-s)}\left[\beta^{\prime}(s)-a \beta(s)\right] d s,
$$

where $b_{1}=b e^{-a \tau}$ and $t \in[-\tau, \infty)$.

Proof. Transforming $x$ by a substitution

$$
x(t)=e^{a t} y(t)
$$

where $y$ is a new unknown function, we obtain

$$
a e^{a t} y(t)+e^{a t} \dot{y}(t)=a e^{a t} y(t)+b e^{a(t-\tau)} y(t-\tau)
$$


or

$$
\dot{y}(t)=b_{1} y(t-\tau)
$$

Correspondingly, the initial condition for (2.17) is

$$
y(t)=e^{-a t} \beta(t), \quad t \in[-\tau, 0]
$$

As follows, from formula (2.7), the solution of the corresponding initial Cauchy problems (2.17), (2.18) is

$$
y(t)=\exp _{\tau}\left\{b_{1}, t\right\} e^{a \tau} \beta(-\tau)+\int_{-\tau}^{0} \exp _{\tau}\left\{b_{1}, t-\tau-s\right\}\left[e^{-a s} \beta^{\prime}(s)-a e^{-a s} \beta(s)\right] d s .
$$

Using substitution (2.15), we obtain

$$
x(t)=\exp _{\tau}\left\{b_{1}, t\right\} e^{a(t+\tau)} \beta(-\tau)+\int_{-\tau}^{0} \exp _{\tau}\left\{b_{1}, t-\tau-s\right\} e^{a(t-s)}\left[\beta^{\prime}(s)-a \beta(s)\right] d s,
$$

which is formula (2.14).

\subsection{First-Order Nonhomogeneous Linear Differential Equations with a Single Delay}

Let a linear non-homogeneous delay equation with a single delay

$$
\dot{x}(t)=a x(t)+b x(t-\tau)+f(t)
$$

be given, where $a, b \in \mathbb{R}$ and $f:[0, \infty) \rightarrow \mathbb{R}$. We consider the Cauchy problem with a zero initial condition

$$
x(t)=0, \quad t \in[-\tau, 0],
$$

that is, we put $\beta \equiv 0$ in (2.6).

Theorem 2.6. The unique solution of the problem (2.21), (2.22) is given by the formula

$$
x(t)=\int_{0}^{t} \exp _{\tau}\left\{b_{1}, t-\tau-s\right\} e^{a(t-s)} f(s) d s,
$$

where $b_{1}=b e^{-a \tau}$.

Proof. We apply substitution (2.15). Then

$$
a e^{a t} y(t)+e^{a t} \dot{y}(t)=a e^{a t} y(t)+b e^{a(t-\tau)} y(t-\tau)+f(t),
$$


or, equivalently,

$$
\dot{y}(t)=b_{1} y(t-\tau)+e^{-a t} f(t) .
$$

We will show that the solution of the non-homogeneous equation (2.25) satisfying a zero initial condition (deduced from (2.15) and (2.22)) is

$$
y(t)=\int_{0}^{t} \exp _{\tau}\left\{b_{1}, t-\tau-s\right\} e^{-a s} f(s) d s
$$

Substituting (2.26) in (2.25), we obtain

$$
\begin{aligned}
& \left.\exp _{\tau}\left\{b_{1}, t-\tau-s\right\} e^{-a s} f(s)\right|_{s=t}+b_{1} \int_{0}^{t} \exp _{\tau}\left\{b_{1}, t-2 \tau-s\right\} e^{-a s} f(s) d s \\
& =b_{1} \int_{0}^{t-\tau} \exp _{\tau}\left\{b_{1}, t-2 \tau-s\right\} e^{-a s} f(s) d s+e^{-a t} f(t) .
\end{aligned}
$$

Since

$$
\left.\exp _{\tau}\left\{b_{1}, t-\tau-s\right\} e^{-a s} f(s)\right|_{s=t}=\exp \left\{b_{1},-\tau\right\} e^{-a t} f(t)=e^{-a t} f(t),
$$

we obtain

$$
\begin{aligned}
& e^{-a t} f(t)+b_{1} \int_{0}^{t-\tau} \exp _{\tau}\left\{b_{1}, t-2 \tau-s\right\} e^{-a s} f(s) d s+b_{1} \int_{t-\tau}^{t} \exp _{\tau}\left\{b_{1}, t-2 \tau-s\right\} e^{-a s} f(s) d s \\
& =b_{1} \int_{0}^{t-\tau} \exp _{\tau}\left\{b_{1}, t-2 \tau-s\right\} e^{-a s} f(s) d s+e^{-a t} f(t) .
\end{aligned}
$$

Hence,

$$
\int_{t-\tau}^{t} \exp _{\tau}\left\{b_{1}, t-2 \tau-s\right\} e^{-a s} f(s) d s=0
$$

This equality is true since

$$
t-2 \tau-s \leq t-2 \tau-(t-\tau)=-\tau
$$

and, by formula (2.1) in Definition 2.1,

$$
\exp _{\tau}\left\{b_{1}, t-2 \tau-s\right\} \equiv 0
$$


if $t-2 \tau-s<-\tau$. In accordance with (2.15), we get

$$
x(t)=e^{a t} y(t)=\int_{0}^{t} \exp _{\tau}\left\{b_{1}, t-\tau-s\right\} e^{a(t-s)} f(s) d s,
$$

that is, formula (2.23) is proved.

Combining Theorems 2.5, and 2.6 we get the following Corollary.

Corollary 2.7. Let the function $\beta$ in (2.6) be continuously differentiable. Then the unique solution of the problems (2.21), (2.6) is given as

$$
\begin{aligned}
x(t)= & \exp _{\tau}\left\{b_{1}, t\right\} e^{a(t+\tau)} \beta(-\tau) \\
& +\int_{-\tau}^{0} \exp _{\tau}\left\{b_{1}, t-\tau-s\right\} e^{a(t-s)}\left[\beta^{\prime}(s)-a \beta(s)\right] d s \\
& +\int_{0}^{t} \exp _{\tau}\left\{b_{1}, t-\tau-s\right\} e^{a(t-s)} f(s) d s,
\end{aligned}
$$

where $b_{1}=b e^{-a \tau}$.

\section{Partial Differential Systems with Delay}

Now we consider second-order autonomous systems of linear partial homogeneous differential equations of parabolic type with a single delay (1.1) where $0 \leq x \leq l$ and $t \geq-\tau$. The initial conditions (1.13), (1.14) are defined for $(x, t) \in[0, l] \times[-\tau, 0]$. Boundary conditions (1.9)-(1.12) are defined for $t \geq-\tau$ and compatibility conditions (1.8) are fulfilled on the interval $-\tau \leq t \leq 0$.

By the transformation

$$
\left(\begin{array}{l}
u(x, t) \\
v(x, t)
\end{array}\right)=S\left(\begin{array}{l}
\xi(x, t) \\
\eta(x, t)
\end{array}\right)
$$

the systems (1.1) can be reduced to a form

$$
\left(\begin{array}{c}
\frac{\partial \xi(x, t)}{\partial t} \\
\frac{\partial \eta(x, t)}{\partial t}
\end{array}\right)=\Lambda\left(\begin{array}{l}
\frac{\partial^{2} \xi(x, t-\tau)}{\partial x^{2}} \\
\frac{\partial^{2} \eta(x, t-\tau)}{\partial x^{2}}
\end{array}\right)+\Sigma\left(\begin{array}{l}
\frac{\partial^{2} \xi(x, t)}{\partial x^{2}} \\
\frac{\partial^{2} \eta(x, t)}{\partial x^{2}}
\end{array}\right)
$$

that is, into two independent scalar equations

$$
\begin{aligned}
& \frac{\partial \xi(x, t)}{\partial t}=\lambda_{1} \frac{\partial^{2} \xi(x, t-\tau)}{\partial x^{2}}+\sigma_{1} \frac{\partial^{2} \xi(x, t)}{\partial x^{2}} \\
& \frac{\partial \eta(x, t)}{\partial t}=\lambda_{2} \frac{\partial^{2} \eta(x, t-\tau)}{\partial x^{2}}+\sigma_{2} \frac{\partial^{2} \eta(x, t)}{\partial x^{2}}
\end{aligned}
$$


Initial and boundary conditions reduce to

$$
\begin{array}{ll}
\xi(0, t)=\mu_{1}^{*}(t), & t \in[-\tau, \infty), \\
\xi(l, t)=\mu_{2}^{*}(t), & t \in[-\tau, \infty), \\
\eta(0, t)=\theta_{1}^{*}(t), & t \in[-\tau, \infty), \\
\eta(l, t)=\theta_{2}^{*}(t), & t \in[-\tau, \infty),
\end{array}
$$

and to

$$
\begin{aligned}
& \xi(x, t)=\varphi^{*}(x, t), \quad(x, t) \in[0, l] \times[-\tau, 0], \\
& \eta(x, t)=\psi^{*}(x, t), \quad(x, t) \in[0, l] \times[-\tau, 0],
\end{aligned}
$$

where

$$
\begin{aligned}
\left(\begin{array}{l}
\mu_{1}^{*}(t) \\
\theta_{1}^{*}(t)
\end{array}\right) & =S^{-1}\left(\begin{array}{l}
\mu_{1}(t) \\
\theta_{1}(t)
\end{array}\right), \\
\left(\begin{array}{l}
\mu_{2}^{*}(t) \\
\theta_{2}^{*}(t)
\end{array}\right) & =S^{-1}\left(\begin{array}{c}
\mu_{2}(t) \\
\theta_{2}(t)
\end{array}\right), \\
\left(\begin{array}{l}
\varphi^{*}(x, t) \\
\psi^{*}(x, t)
\end{array}\right) & =S^{-1}\left(\begin{array}{l}
\varphi(x, t) \\
\psi(x, t)
\end{array}\right) .
\end{aligned}
$$

\subsection{Constructing of a Solution of (3.3)}

We will consider (3.3) with the boundary conditions (3.5), (3.6) and the initial condition (3.7). We will construct a solution in the form

$$
\xi(x, t)=\xi_{0}(x, t)+\xi_{1}(x, t)+\mu_{1}^{*}(t)+\frac{x}{l}\left[\mu_{2}^{*}(t)-\mu_{1}^{*}(t)\right]
$$

where $(x, t) \in[0, l] \times[-\tau, \infty), \xi_{0}(x, t)$ is a solution of (3.3) with zero boundary conditions

$$
\xi_{0}(0, t)=0, \quad \xi_{0}(l, t)=0, \quad t \in[-\tau, \infty)
$$

and with a nonzero initial condition

$$
\xi_{0}(x, t)=\Phi(x, t):=\varphi^{*}(x, t)-\mu_{1}^{*}(t)-\frac{x}{l}\left[\mu_{2}^{*}(t)-\mu_{1}^{*}(t)\right], \quad(x, t) \in[0, l] \times[-\tau, 0],
$$

and $\xi_{1}(x, t)$ is a solution of a non-homogeneous equation

$$
\frac{\partial \xi(x, t)}{\partial t}=\lambda_{1} \frac{\partial^{2} \xi(x, t-\tau)}{\partial x^{2}}+\sigma_{1} \frac{\partial^{2} \xi(x, t)}{\partial x^{2}}+F(x, t),
$$


where

$$
F(x, t):=-\dot{\mu}_{1}^{*}(t)-\frac{x}{l}\left[\dot{\mu}_{2}^{*}(t)-\dot{\mu}_{1}^{*}(t)\right]
$$

with zero boundary conditions

$$
\xi_{1}(0, t)=0, \quad \xi_{1}(l, t)=0, \quad t \in[-\tau, \infty)
$$

and a zero initial condition

$$
\xi_{1}(x, t)=0, \quad(x, t) \in[0, l] \times[-\tau, 0] .
$$

\subsubsection{Equation (3.3)-Solution of the Problems (3.11), (3.12)}

For finding a solution $\xi=\xi_{0}(x, t)$ of (3.3), we will use the method of separation of variables. The solution $\xi_{0}(x, t)$ is seen as the product of two unknown functions $X(x)$ and $T(t)$, that is,

$$
\xi_{0}(x, t)=X(x) T(t) .
$$

Substituting (3.17) into (3.3), we obtain

$$
X(x) T^{\prime}(t)=\lambda_{1} X^{\prime \prime}(x) T(t-\tau)+\sigma_{1} X^{\prime \prime}(x) T(t) .
$$

Separating variables, we have

$$
\frac{T^{\prime}(t)}{\lambda_{1} T(t-\tau)+\sigma_{1} T(t)}=\frac{X^{\prime \prime}(x)}{X(x)}=-\kappa^{2},
$$

where $\kappa$ is a constant. We consider two differential equations

$$
\begin{gathered}
T^{\prime}(t)+\sigma_{1} \kappa^{2} T(t)+\lambda_{1} \kappa^{2} T(t-\tau)=0, \\
X^{\prime \prime}(x)+\kappa^{2} X(x)=0 .
\end{gathered}
$$

Nonzero solutions of (3.21) that satisfy zero boundary conditions

$$
X(0)=0, \quad X(l)=0,
$$


exist for the choice $\kappa^{2}=\kappa_{n}^{2}=(\pi n / l)^{2}, n=1,2, \ldots$, and are defined by the formulas

$$
X(x)=X_{n}(x)=A_{n} \sin \frac{\pi n}{l} x, \quad n=1,2, \ldots,
$$

where $A_{n}$ are arbitrary constants. Now we consider (3.20) with $\kappa=\kappa_{n}$ :

$$
T_{n}^{\prime}(t)=-\sigma_{1}\left(\frac{\pi n}{l}\right)^{2} T_{n}(t)-\lambda_{1}\left(\frac{\pi n}{l}\right)^{2} T_{n}(t-\tau), \quad n=1,2, \ldots
$$

Each of (3.24) represents a linear first-order delay differential equation with constant coefficients. We will specify initial conditions for each of (3.23), (3.24). To obtain such initial conditions, we expand the corresponding initial condition $\Phi(x, t)$ (see (3.12)) into Fourier series

$$
\Phi(x, t)=\sum_{n=1}^{\infty} \Phi_{n}(t) \sin \frac{\pi n}{l} x, \quad(x, t) \in[0, l] \times[-\tau, 0]
$$

where

$$
\begin{aligned}
\Phi_{n}(t)= & \frac{2}{l} \int_{0}^{l} \Phi(s, t) \sin \frac{\pi n}{l} s d s \\
= & \frac{2}{l} \int_{0}^{l}\left[\varphi^{*}(s, t)-\mu_{1}^{*}(t)-\frac{s}{l}\left(\mu_{2}^{*}(t)-\mu_{1}^{*}(t)\right)\right] \sin \frac{\pi n}{l} s d s \\
= & \frac{2}{l} \int_{0}^{l} \varphi^{*}(s, t) \sin \frac{\pi n}{l} s d s \\
& -\frac{2 \mu_{1}^{*}(t)}{l} \int_{0}^{l} \sin \frac{\pi n}{l} s d s-\frac{2\left(\mu_{2}^{*}(t)-\mu_{1}^{*}(t)\right)}{l^{2}} \int_{0}^{l} s \cdot \sin \frac{\pi n}{l} s d s \\
= & \frac{2}{l} \int_{0}^{l} \varphi^{*}(s, t) \sin \frac{\pi n}{l} s d s+\frac{2}{\pi n}\left[(-1)^{n} \mu_{2}^{*}(t)-\mu_{1}^{*}(t)\right], \quad t \in[-\tau, 0] .
\end{aligned}
$$

We will find an analytical solution of the problem (3.24) with initial function (3.26), that is, we will find an analytical solution of the Cauchy initial problem

$$
\begin{gathered}
T_{n}^{\prime}(t)=-\sigma_{1}\left(\frac{\pi n}{l}\right)^{2} T_{n}(t)-\lambda_{1}\left(\frac{\pi n}{l}\right)^{2} T_{n}(t-\tau), \\
T_{n}(t)=\Phi_{n}(t), \quad t \in[-\tau, 0],
\end{gathered}
$$

for every $n=1,2, \ldots$ Using the results of Part 2, we will solve the problem (3.27). According 
to formula (2.14), we get

$$
\begin{aligned}
T_{n}(t)= & \exp _{\tau}\left\{r_{1 n}, t\right\} e^{-\sigma_{1}(\pi n / l)^{2}(t+\tau)} \Phi_{n}(-\tau) \\
& +\int_{-\tau}^{0} \exp _{\tau}\left\{r_{1 n}, t-\tau-s\right\} e^{-\sigma_{1}(\pi n / l)^{2}(t-s)}\left[\Phi_{n}^{\prime}(s)+\sigma_{1}\left(\frac{\pi n}{l}\right)^{2} \Phi_{n}(s)\right] d s,
\end{aligned}
$$

where

$$
r_{1 n}=-\lambda_{1}\left(\frac{\pi n}{l}\right)^{2} e^{\sigma_{1}(\pi n / l)^{2} \tau}, \quad n=1,2, \ldots
$$

Thus, the solution $\xi_{0}(x, t)$ of the homogeneous equation (3.3) that satisfies zero boundary conditions (3.11) and a nonzero initial condition (3.12) (to satisfy (3.12) we set $A_{n}=1, n=$ $1,2, \ldots$ in $(3.23))$ is

$$
\begin{aligned}
\xi_{0}(x, t)=\sum_{n=1}^{\infty} & {\left[\exp _{\tau}\left\{r_{1 n}, t\right\} e^{-\sigma_{1}(\pi n / l)^{2}(t+\tau)} \Phi_{n}(-\tau)\right.} \\
& \left.\quad+\int_{-\tau}^{0} \exp _{\tau}\left\{r_{1 n}, t-\tau-s\right\} e^{-\sigma_{1}(\pi n / l)^{2}(t-s)}\left[\Phi_{n}^{\prime}(s)+\sigma_{1}\left(\frac{\pi n}{l}\right)^{2} \Phi_{n}(s)\right] d s\right] \sin \frac{\pi n}{l} x,
\end{aligned}
$$

where $\Phi_{n}$ is defined by (3.26), $r_{1 n}$ by (3.29), and $(x, t) \in[0, l] \times[-\tau, \infty)$.

\subsubsection{Nonhomogeneous Equation (3.13)}

Further, we will consider the non-homogeneous equation (3.13) with zero boundary conditions (3.15) and a zero initial condition (3.16). We will try to find the solution in the form of an expansion

$$
\xi_{1}(x, t)=\sum_{n=1}^{\infty} T_{n}^{0}(t) \sin \frac{\pi n}{l} x,
$$

where $(x, t) \in[0, l] \times[-\tau, \infty)$ and $T_{n}^{0}:[-\tau, \infty) \rightarrow \mathbb{R}$ are unknown functions. Substituting (3.31) into (3.13) and equating the coefficients of the same functional terms, we will obtain a system of equations:

$$
\left(T_{n}^{0}\right)^{\prime}(t)=-\sigma_{1}\left(\frac{\pi n}{l}\right)^{2} T_{n}^{0}(t)-\lambda_{1}\left(\frac{\pi n}{l}\right)^{2} T_{n}^{0}(t-\tau)+f_{n}(t), \quad t \in[0, \infty), n=1,2, \ldots,
$$


where $f_{n}:[-\tau, \infty) \rightarrow \mathbb{R}$ are Fourier coefficients of the function $F(x, t)$, that is,

$$
\begin{aligned}
f_{n}(t) & =\frac{2}{l} \int_{0}^{l} F(s, t) \sin \frac{\pi n}{l} s d s \\
& =-\frac{2}{l} \int_{0}^{l}\left(\dot{\mu}_{1}^{*}(t)+\frac{s}{l}\left[\dot{\mu}_{2}^{*}(t)-\dot{\mu}_{1}^{*}(t)\right]\right) \sin \frac{\pi n}{l} s d s \\
& =-\frac{2}{l} \dot{\mu}_{1}^{*}(t) \int_{0}^{l} \sin \frac{\pi n}{l} s d s-\frac{2}{l^{2}}\left(\dot{\mu}_{2}^{*}(t)-\dot{\mu}_{1}^{*}(t)\right) \int_{0}^{l} s \cdot \sin \frac{\pi n}{l} s d s \\
& =-\frac{2}{\pi n}\left((-1)^{n+1} \dot{\mu}_{2}^{*}(t)+\dot{\mu}_{1}^{*}(t)\right) .
\end{aligned}
$$

In accordance with (3.15), we assume zero initial conditions

$$
T_{n}^{0}(t)=0, \quad t \in[-\tau, 0], n=1,2, \ldots
$$

for every equation (3.32). Then, by formula (2.23) in Theorem 2.6, a solution of each of the problems (3.32), (3.34) can be written as

$$
T_{n}^{0}(t)=\int_{0}^{t} \exp _{\tau}\left\{r_{1 n}, t-\tau-s\right\} e^{-\sigma_{1}(\pi n / l)^{2}(t-s)} f_{n}(s) d s, \quad t \in[-\tau, \infty), n=1,2, \ldots,
$$

where $r_{1 n}$ is defined by formula (3.29).

Hence, the solution of the non-homogeneous equation (3.13) with zero boundary conditions and a zero initial condition is

$$
\xi_{1}(x, t)=\sum_{n=1}^{\infty}\left[\int_{0}^{t} \exp _{\tau}\left\{r_{1 n}, t-\tau-s\right\} e^{-\sigma_{1}(\pi n / l)^{2}(t-s)} f_{n}(s) d s\right] \sin \frac{\pi n}{l} x,
$$

where $f_{n}$ is given by formula (3.33).

\subsection{Formal Solution of the Boundary Value Problem}

Now we complete the particular results giving a solution of the boundary value problem of the initial system (1.1) satisfying conditions (1.9)-(1.14) in the form of a formal series. Conditions of their convergence will be discussed in the following Part 4.

Since the solutions $\xi_{0}(x, t)$ and $\xi_{1}(x, t)$ of auxiliary problems are formally differentiable once with respect to $t$ and twice with respect to $x$, and functions $\mu_{1}^{*}(t), \mu_{2}^{*}(t)$ are once differentiable, we conclude that a formal solution of the first boundary value problem (3.5), 
(3.6) and (3.7) for (3.3) can be expressed by the formula:

$$
\begin{aligned}
\xi(x, t)=\sum_{n=1}^{\infty} & {\left[\exp _{\tau}\left\{r_{1 n}, t\right\} e^{-\sigma_{1}(\pi n / l)^{2}(t+\tau)} \Phi_{n}(-\tau)\right.} \\
& +\int_{-\tau}^{0} \exp _{\tau}\left\{r_{1 n}, t-\tau-s\right\} e^{-\sigma_{1}(\pi n / l)^{2}(t-s)}\left[\Phi_{n}^{\prime}(s)+\sigma_{1}\left(\frac{\pi n}{l}\right)^{2} \Phi_{n}(s)\right] d s \\
& \left.+\int_{0}^{t} \exp _{\tau}\left\{r_{1 n}, t-\tau-s\right\} e^{-\sigma_{1}(\pi n / l)^{2}(t-s)} f_{n}(s) d s\right] \sin \frac{\pi n}{l} x \\
+ & \mu_{1}^{*}(t)+\frac{x}{l}\left[\mu_{2}^{*}(t)-\mu_{1}^{*}(t)\right]
\end{aligned}
$$

where $(x, t) \in[0, l] \times[-\tau, \infty)$, coefficients $\Phi_{n}$ are defined by formulas (3.26), coefficients $f_{n}$ by formulas (3.33), and the numbers $r_{1 n}$ by formula (3.29).

Similarly, a formal solution of the first boundary value problem for (3.4) is given by the formula:

$$
\begin{aligned}
\eta(x, t)=\sum_{n=1}^{\infty} & {\left[\exp _{\tau}\left\{r_{2 n}, t\right\} e^{-\sigma_{2}(\pi n / l)^{2}(t+\tau)} \Psi_{n}(-\tau)\right.} \\
& +\int_{-\tau}^{0} \exp _{\tau}\left\{r_{2 n}, t-\tau-s\right\} e^{-\sigma_{2}(\pi n / l)^{2}(t-s)}\left[\Psi_{n}^{\prime}(s)+\sigma_{2}\left(\frac{\pi n}{l}\right)^{2} \Psi_{n}(s)\right] d s \\
& \left.+\int_{0}^{t} \exp _{\tau}\left\{r_{2 n}, t-\tau-s\right\} e^{-\sigma_{2}(\pi n / l)^{2}(t-s)} g_{n}(s) d s\right] \sin \frac{\pi n}{l} x \\
+ & \theta_{1}^{*}(t)+\frac{x}{l}\left[\theta_{2}^{*}(t)-\theta_{1}^{*}(t)\right],
\end{aligned}
$$

where $(x, t) \in[0, l] \times[-\tau, \infty)$ and (by analogy with (3.26), (3.33) and (3.29))

$$
\begin{gathered}
\Psi_{n}(t)=\frac{2}{l} \int_{0}^{l} \psi^{*}(s, t) \sin \frac{\pi n}{l} s d s+\frac{2}{\pi n}\left[(-1)^{n} \theta_{2}^{*}(t)-\theta_{1}^{*}(t)\right], \quad t \in[-\tau, 0] \\
g_{n}(t)=-\frac{2}{\pi n}\left((-1)^{n+1} \dot{\theta}_{2}^{*}(t)+\dot{\theta}_{1}^{*}(t)\right), \quad t \in[-\tau, \infty) \\
r_{2 n}=-\lambda_{2}\left(\frac{\pi n}{l}\right)^{2} e^{\sigma_{2}(\pi n / l)^{2} \tau}
\end{gathered}
$$

Then, a formal solution of the boundary value problem of the initial system (1.1) satisfying conditions (1.9)-(1.14) is given by the formulas

$$
\begin{aligned}
& u(x, t)=s_{11} \xi(x, t)+s_{12} \eta(x, t), \\
& v(x, t)=s_{21} \xi(x, t)+s_{22} \eta(x, t),
\end{aligned}
$$

where $\xi(x, t), \eta(x, t)$ are defined by (3.37) and (3.38). 


\section{Convergence of Formal Series}

A solution of the first boundary value problem for (3.3), (3.4) is presented in the form of formal series (3.37), (3.38). We will show that, when certain conditions are satisfied, the series (together with its relevant partial derivatives) converges for $(x, t) \in[0, l] \times\left[-\tau, t^{*}\right]$ where $t^{*}>0$ is arbitrarily large and, consequently, is a solution of partial delay differential equations (3.3), (3.4).

Theorem 4.1. Let, for the functions

$$
\Phi_{n}:[-\tau, 0] \longrightarrow \mathbb{R}, \quad f_{n}:[-\tau, \infty) \longrightarrow \mathbb{R}, \quad n=1,2, \ldots
$$

defined by (3.26) and (3.33), for an integer $k \geq 1$ and arbitrary $t^{*} \in[(k-1) \tau, k \tau]$, there exist constants $M \geq 0, \alpha>0$ such that

$$
e^{-\sigma_{1}(\pi n / l)^{2}\left(t^{*}-(k-1) \tau\right)} n^{2 k} \max _{-\tau \leq t \leq k \tau}\left|f_{n}(t)\right| \leq \frac{M}{n^{1+\alpha}}, \quad e^{-\sigma_{1}(\pi n / l)^{2}\left(t^{*}-(k-1) \tau\right)} n^{2 k} \max _{-\tau \leq t \leq 0}\left|\Phi_{n}(t)\right| \leq \frac{M}{n^{3+\alpha}} .
$$

Then, for $(x, t) \in[0, l] \times[0, k \tau]$, the formal series on the right-hand side of expression (3.37) as well as its first derivative with respect to $t$ and its second derivative with respect to $x$ converge uniformly. Moreover, equality (3.37) holds, and the function $\xi(x, t)$ is a solution of $(3.3)$ for $(x, t) \in[0, l] \times$ $[-\tau, k \tau]$.

Proof. First we prove that the right-hand side of expression (3.37) uniformly converges. Decompose the function $\xi(x, t)$ as

$$
\xi(x, t)=S_{1}(x, t)+S_{2}(x, t)+S_{3}(x, t)+\mu_{1}^{*}(t)+\frac{x}{l}\left[\mu_{2}^{*}(t)-\mu_{1}^{*}(t)\right],
$$

where

$$
\begin{gathered}
S_{1}(x, t)=\sum_{n=1}^{\infty} A_{n}(t) \sin \frac{\pi n}{l} x, \\
A_{n}(t):=\exp _{\tau}\left\{r_{1 n}, t\right\} e^{-\sigma_{1}(\pi n / l)^{2}(t+\tau)} \Phi_{n}(-\tau), \\
S_{2}(x, t)=\sum_{n=1}^{\infty} B_{n}(t) \sin \frac{\pi n}{l} x, \\
B_{n}(t):=\int_{-\tau}^{0} \exp _{\tau}\left\{r_{1 n}, t-\tau-s\right\} e^{-\sigma_{1}(\pi n / l)^{2}(t-s)}\left[\Phi_{n}^{\prime}(s)+\sigma_{1}\left(\frac{\pi n}{l}\right)^{2} \Phi_{n}(s)\right] d s, \\
S_{3}(x, t)=\sum_{n=1}^{\infty} C_{n}(t) \sin \frac{\pi n}{l} x, \\
C_{n}(t):=\int_{0}^{t} \exp _{\tau}\left\{r_{1 n}, t-\tau-s\right\} e^{-\sigma_{1}(\pi n / l)^{2}(t-s)} f_{n}(s) d s .
\end{gathered}
$$


In the following parts, we will prove the uniform convergence of each of the series $S_{i}(x, t)$, $i=1,2,3$ separately.

Throughout the proof, we use the delayed exponential function defined by Definition 2.1, formula (2.1). Note that this function is continuous on $\mathbb{R} \backslash\{-\tau\}$ and at knots $t=k \tau$ where $k=0,1, \ldots$ two lines in formula (2.1) can be applied. We use this property in the proof without any special comment.

\section{Uniform Convergence of the Series $S_{1}(x, t)$}

We consider the coefficients $A_{n}(t), n=1,2, \ldots$ of the first series $S_{1}(x, t)$. As follows from Definition 2.1 of the delayed exponential function, the following equality holds:

$$
\begin{aligned}
A_{n}\left(t^{*}\right)= & \exp _{\tau}\left\{r_{1 n}, t^{*}\right\} e^{-\sigma_{1}(\pi n / l)^{2}\left(t^{*}+\tau\right)} \Phi_{n}(-\tau) \\
=e^{-\sigma_{1}(\pi n / l)^{2}\left(t^{*}+\tau\right)} \Phi_{n}(-\tau) & {\left[1+r_{1 n} \frac{t^{*}}{1 !}+r_{1 n}^{2} \frac{\left(t^{*}-\tau\right)^{2}}{2 !}+\cdots+r_{1 n}^{k} \frac{\left(t^{*}-(k-1) \tau\right)^{k}}{k !}\right] } \\
=e^{-\sigma_{1}(\pi n / l)^{2}\left(t^{*}+\tau\right)} \Phi_{n}(-\tau) & {\left[1-\lambda_{1}\left(\frac{\pi n}{l}\right)^{2} e^{\sigma_{1}(\pi n / l)^{2} \tau} \frac{t^{*}}{1 !}+\lambda_{1}^{2}\left(\frac{\pi n}{l}\right)^{4} e^{2 \sigma_{1}(\pi n / l)^{2} \tau} \frac{\left(t^{*}-\tau\right)^{2}}{2 !}\right.} \\
& \left.+\cdots+\left(-\lambda_{1}\right)^{k}\left(\frac{\pi n}{l}\right)^{2 k} e^{k \sigma_{1}(\pi n / l)^{2} \tau} \frac{\left(t^{*}-(k-1) \tau\right)^{k}}{k !}\right] .
\end{aligned}
$$

Therefore,

$$
\begin{aligned}
S_{1}\left(x, t^{*}\right)= & \sum_{n=1}^{\infty} A_{n}\left(t^{*}\right) \sin \frac{\pi n}{l} x=\sum_{n=1}^{\infty} \exp _{\tau}\left\{r_{1 n}, t^{*}\right\} e^{-\sigma_{1}(\pi n / l)^{2}\left(t^{*}+\tau\right)} \Phi_{n}(-\tau) \sin \frac{\pi n}{l} x \\
= & \sum_{n=1}^{\infty} e^{-\sigma_{1}(\pi n / l)^{2}\left(t^{*}+\tau\right)} \Phi_{n}(-\tau) \sin \frac{\pi n}{l} x-\lambda_{1} \frac{t^{*}}{1 !} \sum_{n=1}^{\infty} e^{-\sigma_{1}(\pi n / l)^{2} t^{*}}\left(\frac{\pi n}{l}\right)^{2} \Phi_{n}(-\tau) \sin \frac{\pi n}{l} x \\
& +\lambda_{1}^{2} \frac{\left(t^{*}-\tau\right)^{2}}{2 !} \sum_{n=1}^{\infty} e^{-\sigma_{1}(\pi n / l)^{2}\left(t^{*}-\tau\right)}\left(\frac{\pi n}{l}\right)^{4} \Phi_{n}(-\tau) \sin \frac{\pi n}{l} x+\cdots \\
& +(-1)^{k} \lambda_{1}^{k} \frac{\left(t^{*}-(k-1) \tau\right)^{k}}{k !} \sum_{n=1}^{\infty} e^{-\sigma_{1}(\pi n / l)^{2}\left(t^{*}-(k-1) \tau\right)}\left(\frac{\pi n}{l}\right)^{2 k} \Phi_{n}(-\tau) \sin \frac{\pi n}{l} x .
\end{aligned}
$$

Due to condition (4.2), we conclude that

$$
e^{-\sigma_{1}(\pi n / l)^{2}\left(t^{*}-(k-1) \tau\right)} n^{2 k}\left|\Phi_{n}(-\tau)\right| \leq \frac{M}{n^{3+\alpha}} .
$$

Therefore, the series $S_{1}\left(x, t^{*}\right)$ converges uniformly with respect to $x \in[0, l]$ and $t^{*} \in[(k-$ 1) $\tau, k \tau]$. If $t^{*} \in\left[\left(k^{*}-1\right) \tau, k^{*} \tau\right]$ where $k^{*} \in\{1,2, \ldots, k-1\}$, then the estimations remain valid. 
Note that inequalities (4.2) are also valid for $k=k^{*}$ because

$$
\begin{gathered}
\max _{-\tau \leq t \leq k^{*} \tau}\left|f_{n}(t)\right| \leq \frac{M}{n^{2 k+1+\alpha}} \leq \frac{M}{n^{2 k^{*}+1+\alpha}}, \\
e^{-\sigma_{1}(\pi n / l)^{2}\left(t^{*}-\left(k^{*}-1\right) \tau\right)} n^{2 k} \max _{-\tau \leq t \leq 0}\left|\Phi_{n}(t)\right| \leq \frac{M}{n^{3+\alpha}} .
\end{gathered}
$$

Consequently, it is easy to see that the series $S_{1}(x, t)$ converges uniformly for $x \in[0, l]$ and $t \in[0, k \tau]$.

\section{Uniform Convergence of the Series $S_{2}(x, t)$}

We consider the coefficients $B_{n}(t), n=1,2, \ldots$ of the second series $S_{2}(x, t)$. In the representation

$$
\begin{aligned}
B_{n}(t)= & \sigma_{1}\left(\frac{\pi n}{l}\right)^{2} \int_{-\tau}^{0} \exp _{\tau}\left\{r_{1 n}, t-\tau-s\right\} e^{-\sigma_{1}(\pi n / l)^{2}(t-s)} \Phi_{n}(s) d s \\
& +\int_{-\tau}^{0} \exp _{\tau}\left\{r_{1 n}, t-\tau-s\right\} e^{-\sigma_{1}(\pi n / l)^{2}(t-s)} \Phi_{n}^{\prime}(s) d s
\end{aligned}
$$

we calculate the second integral by parts and use formula (2.2) in Lemma 2.2:

$$
\begin{aligned}
B_{n}(t)= & \sigma_{1}\left(\frac{\pi n}{l}\right)^{2} \int_{-\tau}^{0} \exp _{\tau}\left\{r_{1 n}, t-\tau-s\right\} e^{-\sigma_{1}(\pi n / l)^{2}(t-s)} \Phi_{n}(s) d s \\
& +\exp _{\tau}\left\{r_{1 n}, t-\tau\right\} e^{-\sigma_{1}(\pi n / l)^{2} t} \Phi_{n}(0)-\exp _{\tau}\left\{r_{1 n}, t\right\} e^{-\sigma_{1}(\pi n / l)^{2}(t+\tau)} \Phi_{n}(-\tau) \\
& +\int_{-\tau}^{0} \lambda_{1}\left(\frac{\pi n}{l}\right)^{2} \exp _{\tau}\left\{r_{1 n}, t-2 \tau-s\right\} e^{-\sigma_{1}(\pi n / l)^{2}(t-s-\tau)} \Phi_{n}(s) d s \\
& -\sigma_{1}\left(\frac{\pi n}{l}\right)^{2} \int_{-\tau}^{0} \exp _{\tau}\left\{r_{1 n}, t-\tau-s\right\} e^{-\sigma_{1}(\pi n / l)^{2}(t-s)} \Phi_{n}(s) d s \\
= & \exp _{\tau}\left\{r_{1 n}, t-\tau\right\} e^{-\sigma_{1}(\pi n / l)^{2} t} \Phi_{n}(0)-\exp _{\tau}\left\{r_{1 n}, t\right\} e^{-\sigma_{1}(\pi n / l)^{2}(t+\tau)} \Phi_{n}(-\tau) \\
& +\int_{-\tau}^{0} \lambda_{1}\left(\frac{\pi n}{l}\right)^{2} \exp _{\tau}\left\{r_{1 n}, t-2 \tau-s\right\} e^{-\sigma_{1}(\pi n / l)^{2}(t-s-\tau)} \Phi_{n}(s) d s \\
= & B_{n 1}(t)-B_{n 2}(t)+B_{n 3}(t),
\end{aligned}
$$

where

$$
\begin{gathered}
B_{n 1}(t)=\exp _{\tau}\left\{r_{1 n}, t-\tau\right\} e^{-\sigma_{1}(\pi n / l)^{2} t} \Phi_{n}(0), \\
B_{n 2}(t)=\exp _{\tau}\left\{r_{1 n}, t\right\} e^{-\sigma_{1}(\pi n / l)^{2}(t+\tau)} \Phi_{n}(-\tau) \\
B_{n 3}(t)=\int_{-\tau}^{0} \lambda_{1}\left(\frac{\pi n}{l}\right)^{2} \exp _{\tau}\left\{r_{1 n}, t-2 \tau-s\right\} e^{-\sigma_{1}(\pi n / l)^{2}(t-s-\tau)} \Phi_{n}(s) d s .
\end{gathered}
$$


As follows from the Definition 2.1 of the delayed exponential function, for $t^{*} \in[(k-1) \tau, k \tau]$, the following equality holds:

$$
\begin{aligned}
B_{n 1}\left(t^{*}\right)= & \exp _{\tau}\left\{r_{1 n}, t^{*}-\tau\right\} e^{-\sigma_{1}(\pi n / l)^{2} t^{*}} \Phi_{n}(0) \\
=e^{-\sigma_{1}(\pi n / l)^{2} t^{*}} \Phi_{n}(0) \times & {\left[1-\lambda_{1}\left(\frac{\pi n}{l}\right)^{2} e^{\sigma_{1}(\pi n / l)^{2} \tau} \frac{t^{*}-\tau}{1 !}+\lambda_{1}^{2}\left(\frac{\pi n}{l}\right)^{4} e^{2 \sigma_{1}(\pi n / l)^{2} \tau} \frac{\left(t^{*}-2 \tau\right)^{2}}{2 !}\right.} \\
& \left.+\cdots+(-1)^{k-1} \lambda_{1}^{k-1}\left(\frac{\pi n}{l}\right)^{2(k-1)} e^{(k-1) \sigma_{1}(\pi n / l)^{2} \tau} \frac{\left(t^{*}-(k-1) \tau\right)^{k-1}}{(k-1) !}\right] .
\end{aligned}
$$

Therefore, for

$$
S_{21}\left(x, t^{*}\right):=\sum_{n=1}^{\infty} B_{n 1}\left(t^{*}\right) \sin \frac{\pi n}{l} x
$$

we get

$$
\begin{aligned}
S_{21}\left(x, t^{*}\right)= & \sum_{n=1}^{\infty} B_{n 1}\left(t^{*}\right) \sin \frac{\pi n}{l} x=\sum_{n=1}^{\infty} \exp _{\tau}\left\{r_{1 n}, t^{*}-\tau\right\} e^{-\sigma_{1}(\pi n / l)^{2} t^{*}} \Phi_{n}(0) \sin \frac{\pi n}{l} x \\
= & \sum_{n=1}^{\infty} e^{-\sigma_{1}(\pi n / l)^{2} t^{*}} \Phi_{n}(0) \sin \frac{\pi n}{l} x-\lambda_{1} \frac{t^{*}-\tau}{1 !} \sum_{n=1}^{\infty}\left(\frac{\pi n}{l}\right)^{2} e^{-\sigma_{1}(\pi n / l)^{2}\left(t^{*}-\tau\right)} \Phi_{n}(0) \sin \frac{\pi n}{l} x \\
& +\lambda_{1}^{2} \frac{\left(t^{*}-2 \tau\right)^{2}}{2 !} \sum_{n=1}^{\infty}\left(\frac{\pi n}{l}\right)^{4} e^{-\sigma_{1}(\pi n / l)^{2}\left(t^{*}-2 \tau\right)} \Phi_{n}(0) \sin \frac{\pi n}{l} x+\cdots \\
& +(-1)^{k-1} \lambda_{1}^{k-1} \frac{\left(t^{*}-(k-1) \tau\right)^{k-1}}{(k-1) !} \sum_{n=1}^{\infty}\left(\frac{\pi n}{l}\right)^{2(k-1)} e^{-\sigma_{1}(\pi n / l)^{2}\left(t^{*}-(k-1) \tau\right)} \Phi_{n}(0) \sin \frac{\pi n}{l} x
\end{aligned}
$$

Due to condition (4.2), we conclude that

$$
e^{-\sigma_{1}(\pi n / l)^{2}\left(t^{*}-(k-1) \tau\right)} n^{2 k}\left|\Phi_{n}(0)\right| \leq \frac{M}{n^{3+\alpha}}
$$

The proof of the uniform convergence of the series $S_{21}(x, t)$ for $x \in[0, l]$ and $t \in[0, k \tau]$ can now be performed in a way similar to the proof of the uniform convergence of the series $S_{1}(x, t)$ for $x \in[0, l]$ and $t \in[0, k \tau]$. 
For the coefficients $B_{n 2}(t)$, the following holds:

$$
\begin{aligned}
B_{n 2}\left(t^{*}\right)= & \exp _{\tau}\left\{r_{1 n}, t^{*}\right\} e^{-\sigma_{1}(\pi n / l)^{2}\left(t^{*}+\tau\right)} \Phi_{n}(-\tau) \\
=e^{-\sigma_{1}(\pi n / l)^{2}\left(t^{*}+\tau\right)} \Phi_{n}(-\tau) \times & {\left[1-\lambda_{1}\left(\frac{\pi n}{l}\right)^{2} e^{\sigma_{1}(\pi n / l)^{2} \tau} \frac{t^{*}}{1 !}+\lambda_{1}^{2}\left(\frac{\pi n}{l}\right)^{4} e^{2 \sigma_{1}(\pi n / l)^{2} \tau} \frac{\left(t^{*}-\tau\right)^{2}}{2 !}\right.} \\
& \left.+\cdots+(-1)^{k} \lambda_{1}^{k}\left(\frac{\pi n}{l}\right)^{2 k} e^{k \sigma_{1}(\pi n / l)^{2} \tau} \frac{\left(t^{*}-(k-1) \tau\right)^{k}}{k !}\right] .
\end{aligned}
$$

Therefore, for

$$
S_{22}\left(x, t^{*}\right):=\sum_{n=1}^{\infty} B_{n 2}\left(t^{*}\right) \sin \frac{\pi n}{l} x
$$

we get

$$
\begin{aligned}
S_{22}\left(x, t^{*}\right)= & \sum_{n=1}^{\infty} B_{n 2}\left(t^{*}\right) \sin \frac{\pi n}{l} x=\sum_{n=1}^{\infty} \exp _{\tau}\left\{r_{1 n}, t^{*}\right\} e^{-\sigma_{1}(\pi n / l)^{2}\left(t^{*}+\tau\right)} \Phi_{n}(-\tau) \sin \frac{\pi n}{l} x \\
= & \sum_{n=1}^{\infty} e^{-\sigma_{1}(\pi n / l)^{2}\left(t^{*}+\tau\right)} \Phi_{n}(-\tau) \sin \frac{\pi n}{l} x-\lambda_{1} \frac{t^{*}}{1 !} \sum_{n=1}^{\infty}\left(\frac{\pi n}{l}\right)^{2} e^{-\sigma_{1}(\pi n / l)^{2} t^{*}} \Phi_{n}(-\tau) \sin \frac{\pi n}{l} x \\
& +\lambda_{1}^{2} \frac{\left(t^{*}-\tau\right)^{2}}{2 !} \sum_{n=1}^{\infty}\left(\frac{\pi n}{l}\right)^{4} e^{-\sigma_{1}(\pi n / l)^{2}\left(t^{*}-\tau\right)} \Phi_{n}(-\tau) \sin \frac{\pi n}{l} x+\cdots \\
& +\lambda_{1}^{k} \frac{\left(t^{*}-(k-1) \tau\right)^{k}}{k !} \sum_{n=1}^{\infty}\left(\frac{\pi n}{l}\right)^{2 k} e^{-\sigma_{1}(\pi n / l)^{2}\left(t^{*}-(k-1) \tau\right)} \Phi_{n}(-\tau) \sin \frac{\pi n}{l} x .
\end{aligned}
$$

Due to (4.7), the proof of the uniform convergence of the series $S_{22}(x, t)$ for $x \in[0, l]$ and $t \in[0, k \tau]$ can now be performed in a way similar to the proof of the uniform convergence of the series $S_{1}(x, t)$ for $x \in[0, l]$ and $t \in[0, k \tau]$.

Finally, we consider the coefficients $B_{n 3}(t)$ at $t=t^{*} \in[(k-1) \tau, k \tau]$. Substituting $t^{*}-$ $2 \tau-s=\omega$, we obtain the following:

$$
\begin{aligned}
B_{n 3}\left(t^{*}\right) & =\int_{-\tau}^{0}\left(\lambda_{1}\left(\frac{\pi n}{l}\right)^{2} e^{\sigma_{1}(\pi n / l)^{2} \tau} \exp _{\tau}\left\{r_{1 n}, t^{*}-2 \tau-s\right\}\right) e^{-\sigma_{1}(\pi n / l)^{2}\left(t^{*}-s\right)} \Phi_{n}(s) d s \\
& =\lambda_{1}\left(\frac{\pi n}{l}\right)^{2} e^{\sigma_{1}(\pi n / l)^{2} \tau} \int_{t^{*}-2 \tau}^{t^{*}-\tau} \exp _{\tau}\left\{r_{1 n}, \omega\right\} e^{-\sigma_{1}(\pi n / l)^{2}(\omega+2 \tau)} \Phi_{n}\left(t^{*}-2 \tau-\omega\right) d \omega .
\end{aligned}
$$


We will split this integral in two:

$$
\begin{aligned}
B_{n 3}\left(t^{*}\right)= & \lambda_{1}\left(\frac{\pi n}{l}\right)^{2} e^{\sigma_{1}(\pi n / l)^{2} \tau} \int_{t^{*}-2 \tau}^{(k-2) \tau} \exp _{\tau}\left\{r_{1 n}, \omega\right\} e^{-\sigma_{1}(\pi n / l)^{2}(\omega+2 \tau)} \Phi_{n}\left(t^{*}-2 \tau-\omega\right) d \omega \\
& +\lambda_{1}\left(\frac{\pi n}{l}\right)^{2} e^{\sigma_{1}(\pi n / l)^{2} \tau} \int_{(k-2) \tau}^{t^{*}-\tau} \exp _{\tau}\left\{r_{1 n}, \omega\right\} e^{-\sigma_{1}(\pi n / l)^{2}(\omega+2 \tau)} \Phi_{n}\left(t^{*}-2 \tau-\omega\right) d \omega .
\end{aligned}
$$

Therefore, owing to the mean value theorem, there are values $\omega_{1}$ and $\omega_{2}$ such that

$$
\begin{gathered}
t^{*}-2 \tau \leq \omega_{1} \leq(k-2) \tau, \\
(k-2) \tau \leq \omega_{2} \leq t^{*}-\tau,
\end{gathered}
$$

and (using the Definition 2.1 of the delayed exponential function) we have

$$
\begin{aligned}
& B_{n 3}\left(t^{*}\right)=\lambda_{1}\left(\frac{\pi n}{l}\right)^{2} e^{-\sigma_{1}(\pi n / l)^{2}\left(\omega_{1}+\tau\right)} \Phi_{n}\left(t^{*}-2 \tau-\omega_{1}\right)\left(k \tau-t^{*}\right) \exp _{\tau}\left\{r_{1 n}, \omega_{1}\right\} \\
&+\lambda_{1}\left(\frac{\pi n}{l}\right)^{2} e^{-\sigma_{1}(\pi n / l)^{2}\left(\omega_{2}+\tau\right)} \Phi_{n}\left(t^{*}-2 \tau-\omega_{2}\right)\left(t^{*}-(k-1) \tau\right) \exp _{\tau}\left\{r_{1 n}, \omega_{2}\right\} \\
&=\lambda_{1}\left(\frac{\pi n}{l}\right)^{2} e^{-\sigma_{1}(\pi n / l)^{2}\left(\omega_{1}+\tau\right)} \Phi_{n}\left(t^{*}-2 \tau-\omega_{1}\right)\left(k \tau-t^{*}\right) \\
& \times {\left[1-\lambda_{1}\left(\frac{\pi n}{l}\right)^{2} e^{\sigma_{1}(\pi n / l)^{2} \tau} \frac{\omega_{1}}{1 !}+\lambda_{1}^{2}\left(\frac{\pi n}{l}\right)^{4} e^{2 \sigma_{1}(\pi n / l)^{2} \tau} \frac{\left(\omega_{1}-\tau\right)^{2}}{2 !}\right.} \\
&\left.+\cdots+(-1)^{k-2} \lambda_{1}^{k-2}\left(\frac{\pi n}{l}\right)^{2(k-2)} e^{(k-2) \sigma_{1}(\pi n / l)^{2} \tau} \frac{\left(\omega_{1}-(k-3) \tau\right)^{k-2}}{(k-2) !}\right] \\
&+ \lambda_{1}\left(\frac{\pi n}{l}\right)^{2} e^{-\sigma_{1}(\pi n / l)^{2}\left(\omega_{2}+\tau\right)} \Phi_{n}\left(t^{*}-2 \tau-\omega_{2}\right)\left(t^{*}-(k-1) \tau\right) \\
& \times {\left[1-\lambda_{1}\left(\frac{\pi n}{l}\right)^{2} e^{\sigma_{1}(\pi n / l)^{2} \tau} \frac{\omega_{2}}{1 !}+\lambda_{1}^{2}\left(\frac{\pi n}{l}\right)^{4} e^{2 \sigma_{1}(\pi n / l)^{2} \tau} \frac{\left(\omega_{2}-\tau\right)^{2}}{2 !}\right.} \\
&\left.+\cdots+(-1)^{k-1} \lambda_{1}^{k-1}\left(\frac{\pi n}{l}\right)^{2(k-1)} e^{(k-1) \sigma_{1}(\pi n / l)^{2} \tau} \frac{\left(\omega_{2}-(k-2) \tau\right)^{k-1}}{(k-1) !}\right] .
\end{aligned}
$$

Hence, for

$$
S_{23}\left(x, t^{*}\right):=\sum_{n=1}^{\infty} B_{n 3}\left(t^{*}\right) \sin \frac{\pi n}{l} x
$$


we get

$$
\begin{aligned}
S_{23}\left(x, t^{*}\right)=\sum_{n=1}^{\infty} B_{n 3}\left(t^{*}\right) \sin \frac{\pi n}{l} x & \\
=\sum_{n=1}^{\infty}\left\{\lambda_{1}\left(\frac{\pi n}{l}\right)^{2} e^{-\sigma_{1}(\pi n / l)^{2}\left(\omega_{1}+\tau\right)} \Phi_{n}\right. & \\
\times & \times\left(t^{*}-2 \tau-\omega_{1}\right)\left(k \tau-t^{*}\right)\left[1-\lambda_{1}\left(\frac{\pi n}{l}\right)^{2} e^{\sigma_{1}(\pi n / l)^{2} \tau} \frac{\omega_{1}}{1 !}+\lambda_{1}^{2}\left(\frac{\pi n}{l}\right)^{4} e^{2 \sigma_{1}(\pi n / l)^{2} \tau}\right. \\
& \times \frac{\left(\omega_{1}-\tau\right)^{2}}{2 !}+\cdots+(-1)^{k-2} \lambda_{1}^{k-2}\left(\frac{\pi n}{l}\right)^{2(k-2)} \\
+ & \lambda_{1}\left(\frac{\pi n}{l}\right)^{2} e^{-\sigma_{1}(\pi n / l)^{2}\left(\omega_{2}+\tau\right)} \Phi_{n}\left(t^{*}-2 \tau-\omega_{2}\right)\left(t^{*}-(k-1) \tau\right) \\
& \quad\left[1-\lambda_{1}\left(\frac{\pi n}{l}\right)^{2} e^{\sigma_{1}(\pi n / l)^{2} \tau} \frac{\omega_{2}}{1 !}+\lambda_{1}^{2}\left(\frac{\pi n}{l}\right)^{4} e^{2 \sigma_{1}(\pi n / l)^{2} \tau} \frac{\left(\omega_{2}-\tau\right)^{2}}{2 !}+\cdots\right. \\
& \left.\left.\quad+(-1)^{k-1} \lambda_{1}^{k-1}\left(\frac{\pi n}{l}\right)^{2(k-1)} e^{(k-1) \sigma_{1}(\pi n / l)^{2} \tau} \frac{\left(\omega_{2}-(k-2) \tau\right)^{k-1}}{(k-1) !}\right]\right\} \sin \frac{\pi n}{l} x .
\end{aligned}
$$

After some rearranging, we get

$$
\begin{aligned}
S_{23}\left(x, t^{*}\right)=\lambda_{1} \sum_{n=1}^{\infty}\left[e^{-\sigma_{1}(\pi n / l)^{2}\left(\omega_{1}+\tau\right)} \Phi_{n}\left(t^{*}-2 \tau-\omega_{1}\right)\left(k \tau-t^{*}\right)\right. & \\
+ & \left.e^{-\sigma_{1}(\pi n / l)^{2}\left(\omega_{2}+\tau\right)} \Phi_{n}\left(t^{*}-2 \tau-\omega_{2}\right)\left(t^{*}-(k-1) \tau\right)\right]\left(\frac{\pi n}{l}\right)^{2} \sin \frac{\pi n}{l} x \\
-\lambda_{1}^{2} \sum_{n=1}^{\infty}\left[e^{-\sigma_{1}(\pi n / l)^{2} \omega_{1}} \Phi_{n}\left(t^{*}-2 \tau-\omega_{1}\right)\left(k \tau-t^{*}\right) \times \frac{\omega_{1}}{1 !}\right. & \left.\quad+e^{-\sigma_{1}(\pi n / l)^{2} \omega_{2}} \Phi_{n}\left(t^{*}-2 \tau-\omega_{2}\right)\left(t^{*}-(k-1) \tau\right) \frac{\omega_{2}}{1 !}\right]\left(\frac{\pi n}{l}\right)^{4} \sin \frac{\pi n}{l} x \\
+\lambda_{1}^{3} \sum_{n=1}^{\infty}\left[e^{-\sigma_{1}(\pi n / l)^{2}\left(\omega_{1}-\tau\right)} \Phi_{n}\left(t^{*}-2 \tau-\omega_{1}\right)\left(k \tau-t^{*}\right) \frac{\left(\omega_{1}-\tau\right)^{2}}{2 !}\right. & \left.\quad+e^{-\sigma_{1}(\pi n / l)^{2}\left(\omega_{2}-\tau\right)} \Phi_{n}\left(t^{*}-2 \tau-\omega_{2}\right)\left(t^{*}-(k-1) \tau\right) \frac{\left(\omega_{2}-\tau\right)^{2}}{2 !}\right]\left(\frac{\pi n}{l}\right)^{6} \sin \frac{\pi n}{l} x
\end{aligned}
$$$$
+\cdots
$$ 
Abstract and Applied Analysis

$$
\begin{aligned}
& +(-1)^{k-2} \lambda_{1}^{k-1} \sum_{n=1}^{\infty}\left[e^{-\sigma_{1}(\pi n / l)^{2}\left(\omega_{1}-(k-3) \tau\right)} \Phi_{n}\left(t^{*}-2 \tau-\omega_{1}\right)\left(k \tau-t^{*}\right)\right. \\
& \times \frac{\left(\omega_{1}-(k-3) \tau\right)^{k-2}}{(k-2) !}+e^{-\sigma_{1}(\pi n / l)^{2}\left(\omega_{2}-(k-3) \tau\right)} \Phi_{n}\left(t^{*}-2 \tau-\omega_{2}\right) \\
& \left.\times\left(t^{*}-(k-1) \tau\right) \frac{\left(\omega_{2}-(k-3) \tau\right)^{k-2}}{(k-2) !}\right]\left(\frac{\pi n}{l}\right)^{2 k-2} \sin \frac{\pi n}{l} x \\
& +(-1)^{k-1} \lambda_{1}^{k}\left(t^{*}-(k-1) \tau\right) \frac{\left(\omega_{2}-(k-2) \tau\right)^{k-1}}{(k-1) !} \\
& \times \sum_{n=1}^{\infty}\left(\frac{\pi n}{l}\right)^{2 k} e^{-\sigma_{1}(\pi n / l)^{2}\left(\omega_{2}-(k-2) \tau\right)} \Phi_{n}\left(t^{*}-2 \tau-\omega_{2}\right) \sin \frac{\pi n}{l} x .
\end{aligned}
$$

Due to condition (4.2), we conclude that

$$
e^{-\sigma_{1}(\pi n / l)^{2}\left(\omega_{2}-(k-2) \tau\right)} n^{2 k} \max _{-\tau \leq t \leq 0}\left|\Phi_{n}(t)\right| \leq \frac{M}{n^{3+\alpha}} .
$$

The proof of the uniform convergence of the series $S_{23}(x, t)$ for $x \in[0, l]$ and $t \in[0, k \tau]$ can now be performed in a way similar to the proof of uniform convergence of the series $S_{1}(x, t)$ for $x \in[0, l]$ and $t \in[0, k \tau]$.

\section{Uniform Convergence of the Series $S_{3}(x, t)$}

We will consider the coefficients $C_{n}(t), n=1,2, \ldots$ of the series $S_{3}(x, t)$ at $t=t^{*} \in[(k-1) \tau, k \tau]$. Substituting $t^{*}-\tau-s=\omega$, we obtain

$$
\begin{aligned}
C_{n}\left(t^{*}\right)= & \int_{0}^{t^{*}} \exp _{\tau}\left\{r_{1 n}, t^{*}-\tau-s\right\} e^{-\sigma_{1}(\pi n / l)^{2}\left(t^{*}-s\right)} f_{n}(s) d s \\
= & \int_{-\tau}^{t^{*}-\tau} \exp _{\tau}\left\{r_{1 n}, \omega\right\} e^{-\sigma_{1}(\pi n / l)^{2}(\omega+\tau)} f_{n}\left(t^{*}-\tau-\omega\right) d \omega \\
= & \int_{-\tau}^{0} \exp _{\tau}\left\{r_{1 n}, \omega\right\} e^{-\sigma_{1}(\pi n / l)^{2}(\omega+\tau)} f_{n}\left(t^{*}-\tau-\omega\right) d \omega \\
& +\int_{0}^{\tau} \exp _{\tau}\left\{r_{1 n}, \omega\right\} e^{-\sigma_{1}(\pi n / l)^{2}(\omega+\tau)} f_{n}\left(t^{*}-\tau-\omega\right) d \omega \\
& +\int_{\tau}^{2 \tau} \exp _{\tau}\left\{r_{1 n}, \omega\right\} e^{-\sigma_{1}(\pi n / l)^{2}(\omega+\tau)} f_{n}\left(t^{*}-\tau-\omega\right) d \omega \\
& +\cdots+\int_{(k-2) \tau}^{t^{*}-\tau} \exp _{\tau}\left\{r_{1 n}, \omega\right\} e^{-\sigma_{1}(\pi n / l)^{2}(\omega+\tau)} f_{n}\left(t^{*}-\tau-\omega\right) d \omega .
\end{aligned}
$$


Owing to the mean value theorem, there are values $\omega_{i}, i=1,2, \ldots, k$ such that

$$
\begin{aligned}
&-\tau \leq \omega_{1} \leq 0,0 \leq \omega_{2} \leq \tau, \ldots,(k-2) \tau \leq \omega_{k} \leq t^{*}-\tau \\
& C_{n}\left(t^{*}\right)= \tau e^{-\sigma_{1}(\pi n / l)^{2}\left(\omega_{1}+\tau\right)} f_{n}\left(t^{*}-\tau-\omega_{1}\right) \\
&+\tau\left[1-\lambda_{1}\left(\frac{\pi n}{l}\right)^{2} e^{\sigma_{1}(\pi n / l)^{2} \tau} \frac{\omega_{2}}{1 !}\right] e^{-\sigma_{1}(\pi n / l)^{2}\left(\omega_{2}+\tau\right)} f_{n}\left(t^{*}-\tau-\omega_{2}\right) \\
&+\tau\left[1-\lambda_{1}\left(\frac{\pi n}{l}\right)^{2} e^{\sigma_{1}(\pi n / l)^{2} \tau} \frac{\omega_{3}}{1 !}+\lambda_{1}^{2}\left(\frac{\pi n}{l}\right)^{4} e^{2 \sigma_{1}(\pi n / l)^{2} \tau} \frac{\left(\omega_{3}-\tau\right)^{2}}{2 !}\right] e^{-\sigma_{1}(\pi n / l)^{2}\left(\omega_{3}+\tau\right)} \\
& \times f_{n}\left(t^{*}-\tau-\omega_{3}\right)+\cdots+\tau\left[1-\lambda_{1}\left(\frac{\pi n}{l}\right)^{2} e^{\sigma_{1}(\pi n / l)^{2} \tau} \frac{\omega_{k-1}}{1 !}+\ldots\right. \\
& \quad+(-1)^{k-2} \lambda_{1}^{k-2}\left(\frac{\pi n}{l}\right)^{2(k-2)} e^{(k-2) \sigma_{1}(\pi n / l)^{2} \tau} \\
&\left.\times \frac{\left[\omega_{k-1}-(k-3) \tau\right]^{k-2}}{(k-2) !}\right] e^{-\sigma_{1}(\pi n / l)^{2}\left(\omega_{k-1}+\tau\right)} \\
&+\left[t_{n}^{*}-(k-1) \tau\right]\left[1-\lambda_{1}\left(\frac{\pi n}{l}\right)^{2} e^{\sigma_{1}(\pi n / l)^{2} \tau} \frac{\omega_{k}}{1 !}+\ldots+(-1)^{k-1}\right. \\
& \times e^{-\sigma_{1}(\pi n / l)^{2}\left(\omega_{k}+\tau\right)} f_{n}\left(t^{*}-\tau-\omega_{k}\right) . \\
&\left.\times \lambda_{1}^{k-1}\left(\frac{\pi n}{l}\right)^{2(k-1)} e^{(k-1) \sigma_{1}(\pi n / l)^{2} \tau} \frac{\left[\omega_{k}-(k-2) \tau\right]^{k-1}}{(k-1) !}\right]
\end{aligned}
$$

Hence,

$$
\begin{gathered}
S_{3}\left(x, t^{*}\right)=\sum_{n=1}^{\infty} C_{n}\left(t^{*}\right) \sin \frac{\pi n}{l} x \\
=\sum_{n=1}^{\infty}\left[\tau \sum_{i=1}^{k-1} e^{-\sigma_{1}(\pi n / l)^{2}\left(\omega_{i}+\tau\right)} f_{n}\left(t^{*}-\tau-\omega_{i}\right)+\left(t^{*}-(k-1) \tau\right)\right. \\
\left.\quad \times e^{-\sigma_{1}(\pi n / l)^{2}\left(\omega_{k}+\tau\right)} f_{n}\left(t^{*}-\tau-\omega_{k}\right)\right] \times \sin \frac{\pi n}{l} x \\
-\lambda_{1} \sum_{n=1}^{\infty}\left[\tau \sum_{i=2}^{k-1} \frac{\omega_{i}}{1 !} e^{-\sigma_{1}(\pi n / l)^{2} \omega_{i}} f_{n}\left(t^{*}-\tau-\omega_{i}\right)+\left(t^{*}-(k-1) \tau\right)\right. \\
\left.\quad \times \frac{\omega_{k}}{1 !} e^{-\sigma_{1}(\pi n / l)^{2} \omega_{k}} f_{n}\left(t^{*}-\tau-\omega_{k}\right)\right]
\end{gathered}
$$




$$
\begin{aligned}
& \times\left(\frac{\pi n}{l}\right)^{2} \sin \frac{\pi n}{l} x \\
& +\lambda_{1}^{2} \sum_{n=1}^{\infty}\left[\tau \sum_{i=3}^{k-1} \frac{\left(\omega_{i}-\tau\right)^{2}}{2 !} e^{-\sigma_{1}(\pi n / l)^{2}\left(\omega_{i}-\tau\right)} f_{n}\left(t^{*}-\tau-\omega_{i}\right)\right. \\
& \left.\quad+\left(t^{*}-(k-1) \tau\right) \frac{\left(\omega_{k}-\tau\right)^{2}}{2 !} e^{-\sigma_{1}(\pi n / l)^{2}\left(\omega_{k}-\tau\right)} f_{n}\left(t^{*}-\tau-\omega_{k}\right)\right] \\
& \times\left(\frac{\pi n}{l}\right)^{4} \sin \frac{\pi n}{l} x+\ldots+(-1)^{k-2} \lambda_{1}^{k-2} \\
& \times \sum_{n=1}^{\infty}\left[\tau \frac{\left(\omega_{k-1}-(k-3) \tau\right)^{k-2}}{(k-2) !} e^{-\sigma_{1}(\pi n / l)^{2}\left(\omega_{k-1}-(k-3) \tau\right)} f_{n}\left(t^{*}-\tau-\omega_{k-1}\right)\right. \\
& \left.\quad+\left[t^{*}-(k-1) \tau\right] \frac{\left(\omega_{k}-(k-3) \tau\right)^{k-2}}{(k-2) !} e^{-\sigma_{1}(\pi n / l)^{2}\left(\omega_{k}-(k-3) \tau\right)} f_{n}\left(t^{*}-\tau-\omega_{k}\right)\right] \\
& \times\left(\frac{\pi n}{l}\right)^{2(k-2)} \sin \frac{\pi n}{l} x \\
& \times \sum_{n=1}^{\infty}\left(\frac{\pi n}{l}\right)^{2(k-1)} e^{-\sigma_{1}(\pi n / l)^{2}\left(\omega_{k}-(k-2) \tau\right)} f_{n}\left(t^{*}-\tau-\omega_{k}\right) \sin \frac{\pi n}{l} x .
\end{aligned}
$$

Due to condition (4.2), we conclude that

$$
e^{-\sigma_{1}(\pi n / l)^{2}\left(\omega_{k}-(k-2) \tau\right)} n^{2(k-1)} \max _{-\tau \leq t \leq k \tau}\left|f_{n}(t)\right| \leq \frac{M}{n^{3+\alpha}} .
$$

$S_{3}(x, t) x \in[0, l] t \in[0, k \tau] S_{1}(x, t) x \in[0, l] t \in[0, k \tau]$.

\section{Uniform Convergence of the Formal Series for $\xi(x, t)$}

Above, the absolute and uniform convergence of the series $S_{1}(x, t), S_{2}(x, t), S_{3}(x, t)$ was proved. Therefore, the series for $\xi(x, t)$ converges absolutely and uniformly as well.

\section{Uniform Convergence of the Formal Series for $\xi_{t}^{\prime}(x, t)$ and $\xi_{x x}^{\prime \prime}(x, t)$}

To prove the uniform convergence of the series for $\xi_{t}^{\prime}(x, t)$ and $\xi_{x x}^{\prime \prime}(x, t)$, we can proceed as in the above proof of the uniform convergence of $\xi(x, t)$. The above scheme can be repeated, and the final inequalities (4.7), (4.15), (4.26), and (4.30) are replaced as follows: inequality (4.7) by

$$
e^{-\sigma_{1}(\pi n / l)^{2}\left(t^{*}-(k-1) \tau\right)} n^{2 k+2}\left|\Phi_{n}(-\tau)\right| \leq \frac{M}{n^{1+\alpha}}
$$


inequality (4.15) by

$$
e^{-\sigma_{1}(\pi n / l)^{2}\left(t^{*}-(k-1) \tau\right)} n^{2 k+2}\left|\Phi_{n}(0)\right| \leq \frac{M}{n^{1+\alpha}}
$$

inequality (4.26) by

$$
e^{-\sigma_{1}(\pi n / l)^{2}\left(\omega_{2}-(k-2) \tau\right)} n^{2 k+2} \max _{-\tau \leq t \leq 0}\left|\Phi_{n}(t)\right| \leq \frac{M}{n^{1+\alpha}}
$$

and inequality (4.30) by

$$
e^{-\sigma_{1}(\pi n / l)^{2}\left(\omega_{k}-(k-2) \tau\right)} n^{2 k} \max _{-\tau \leq t \leq k \tau}\left|f_{n}(t)\right| \leq \frac{M}{n^{1+\alpha}} .
$$

The proof of the uniform convergence of the series, which represents the solution $\eta(x, t)$ by formula (3.38), and the proof of the uniform convergence of the series for $\eta_{x}^{\prime}(x, t)$ and $\eta_{x x}^{\prime \prime}(x, t)$ are much the same.

Corollary 4.2. Functions $u(x, t), v(x, t)$ are linear combinations of $\xi(x, t)$ and $\eta(x, t)$. Therefore, representations (3.40) are the solutions of the system system (1.1) satisfying all boundary and initial conditions (1.9)-(1.14).

Remark 4.3. Tracing the proof of Theorem 4.1, we see that inequalities (4.2) for functions $\Phi_{n}$ : $[-\tau, 0] \rightarrow \mathbb{R}, n=1,2, \ldots$, and $f_{n}:[-\tau, \infty) \rightarrow \mathbb{R}, n=1,2, \ldots$ are too restrictive if $t^{*} \in$ $\left[\left(k^{*}-1\right) \tau,\left(k^{*}-1\right) \tau+\varepsilon\right], k^{*} \in\{1,2, \ldots, k\}$, where $\varepsilon$ is an arbitrarily small positive number. The question whether the series are uniformly convergent for $(x, t) \in[0, l] \times\left[\left(k^{*}-1\right) \tau,\left(k^{*}-1\right) \tau+\varepsilon\right]$, $k^{*} \in\{1,2, \ldots, k\}$ remains open if, for example, $\Phi_{n}:[-\tau, 0] \rightarrow \mathbb{R}, n=1,2, \ldots$, and $f_{n}$ : $[-\tau, \infty) \rightarrow \mathbb{R}, n=1,2, \ldots$ satisfy only the inequalities

$$
\max _{-\tau \leq t \leq k \tau}\left|f_{n}(t)\right| \leq \frac{M^{*}}{n}, \quad \max _{-\tau \leq t \leq 0}\left|\Phi_{n}(t)\right| \leq \frac{M^{*}}{n}
$$

for a positive constant $M^{*}$ because inequalities (4.2) cannot be valid. Nevertheless, in such a case, the series converge at least point-wise for $t \in\left[\left(k^{*}-1\right) \tau,\left(k^{*}-1\right) \tau+\varepsilon\right], k^{*} \in\{1,2, \ldots, k\}$ and uniformly for $x \in[0, l]$. In other words, in such a case, the series converge uniformly for

$$
(x, t) \in[0, l] \times\left([0, k \tau] \backslash \bigcup_{k^{*}=1}^{k}\left[\left(k^{*}-1\right) \tau,\left(k^{*}-1\right) \tau+\varepsilon\right]\right),
$$

(where $\varepsilon>0$ is fixed but arbitrarily small).

\section{Acknowledgments}

The paper was supported by the Grants P201/10/1032 and P201/11/0768 of the Czech Grant Agency (Prague), by the Council of Czech Government Grant MSM 00216 30519, and by 
the Grant FEKT-S-11-2-921 of Faculty of Electrical Engineering and Communication, Brno, University of Technology.

\section{References}

[1] I. G. Petrovsky, Lectures on Partial Differential Equations, Dover Publications, New York, NY, USA, 1991, Translated from the Russian by A. Shenitzer, reprint of the 1964 English translation.

[2] E. Kamke, Spravochnik po obyknovennym differentsial'nym uravneniyam, Izdat. Nauka, Moscow, Russia, 1971, Supplemented by a translation by N. H. Rozov of excerpts of articles by D. S. Mitrinović and J. Zbornik. Fourth revised edition. Translated from the sixth German edition and revised in accordance with the eighth German edition by S.V. Fomin.

[3] A. D. Polyanin, Handbook of Linear Partial Differential Equations for Engineers and Scientists, Chapman \& Hall/CRC Press, Boca Raton, Fla, USA, 2002.

[4] A. D. Polyanin and V. F. Zaitsev, Handbook of Exact Solutions for Ordinary Differential Equations, Chapman \& Hall/CRC Press, Boca Raton, Fla, USA, 2nd edition, 2003.

[5] B. L. Rozhdestvenskii and N. N. Yanenko, Systems of Quasilinear Equations and Their Applications to Gas Dynamics, vol. 55 of Translations of Mathematical Monographs, American Mathematical Society, Providence, RI, USA, 1983, Translated from the Russian by J. R. Schulenberger.

[6] F. R. Gantmacher, The Theory of Matrices, vol. 1, AMS Chelsea Publishing, Providence, RI, USA, 2000, Translated from the Russian by K. A. Hirsch, reprint of the 1959 translation.

[7] A. Boichuk, J. Diblík, D. Khusainov, and M. Růžičková, “Fredholm's boundary-value problems for differential systems with a single delay," Nonlinear Analysis, vol. 72, no. 5, pp. 2251-2258, 2010.

[8] A. Boichuk, J. Diblík, D. Khusainov, and M. Růžičková, "Boundary value problems for delay differential systems," Advances in Difference Equations, vol. 210, Article ID 593834, 2010.

[9] A. Boichuk, J. Diblík, D. Khusainov, and M. Růžičková, "Boundary-value problems for weakly nonlinear delay differential systems," Abstract and Applied Analysis, vol. 2011, Article ID 631412, 19 pages, 2011.

[10] J. Diblík, D. Khusainov, Ya. Lukáčová, and M. Rưžičková, "Representation of the solution of the Cauchy problem for an oscillatory system with pure delay," Nelinijni Kolyvannya, vol. 11, no. 2, pp. 261-270, 2008 (Russian), English translation: J. Diblík, D. Khusainov, J. Lukáčová and M. Růžičková, "Representation of a solution of the Cauchy problem for an oscillating system with pure delay", Nonlinear Oscillations, vol. 11, no. 2, pp. 276-285, 2008. 


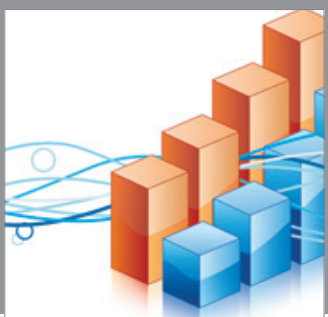

Advances in

Operations Research

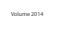

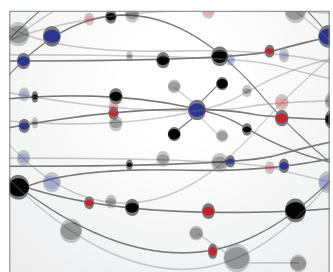

\section{The Scientific} World Journal
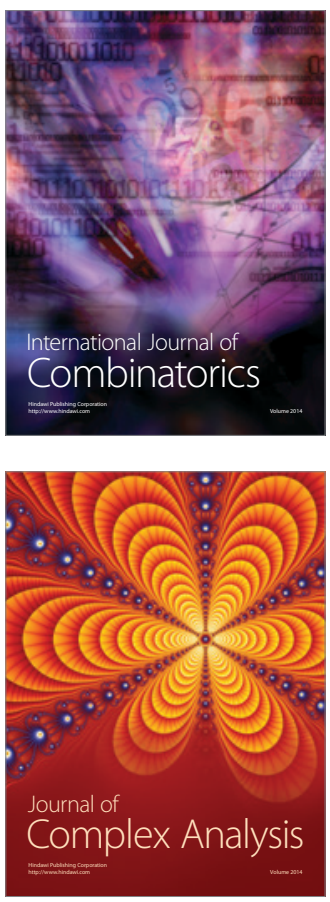

International Journal of

Mathematics and

Mathematical

Sciences
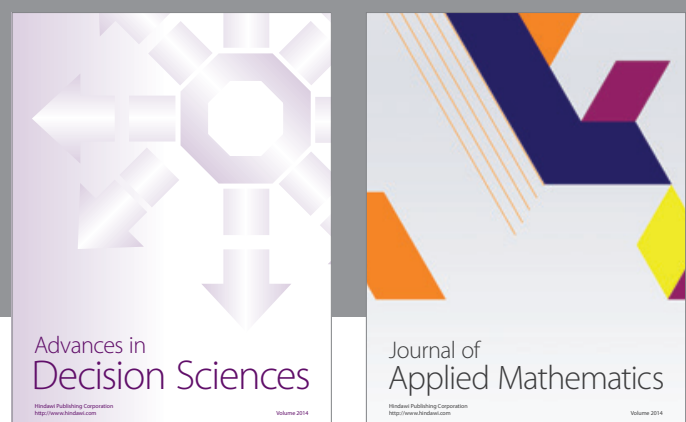

Journal of

Applied Mathematics
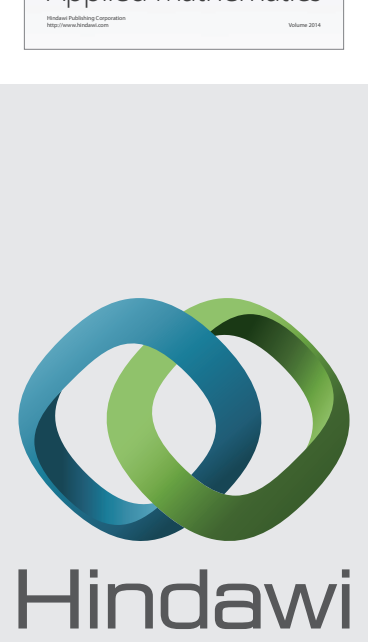

Submit your manuscripts at http://www.hindawi.com
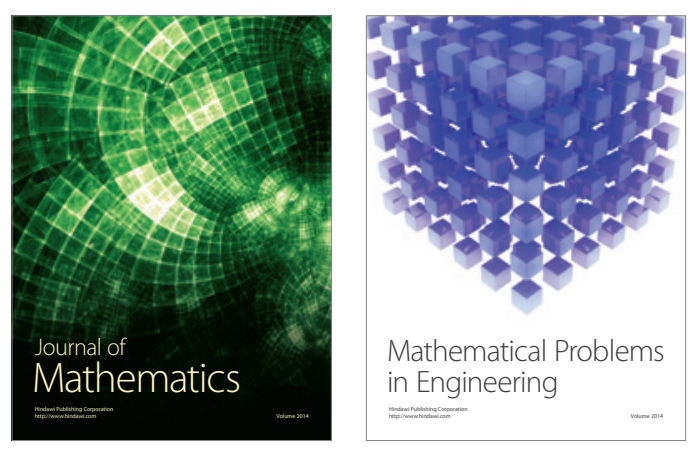

Mathematical Problems in Engineering
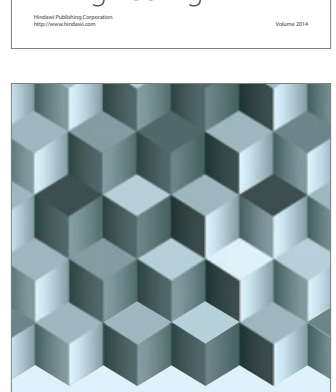

Journal of

Function Spaces
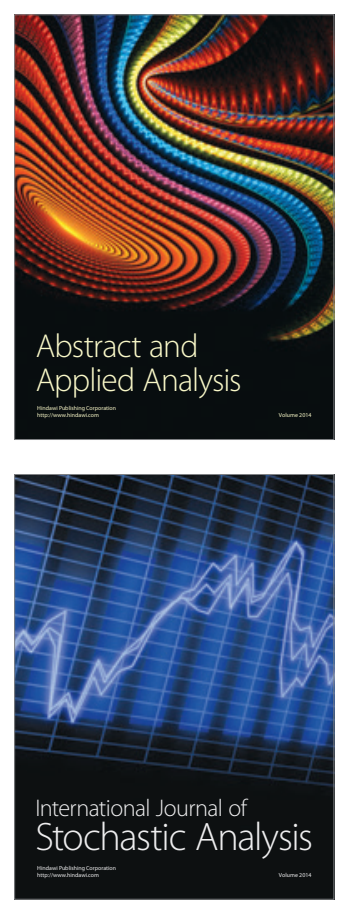

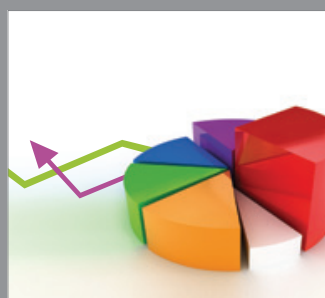

ournal of

Probability and Statistics

Promensencen
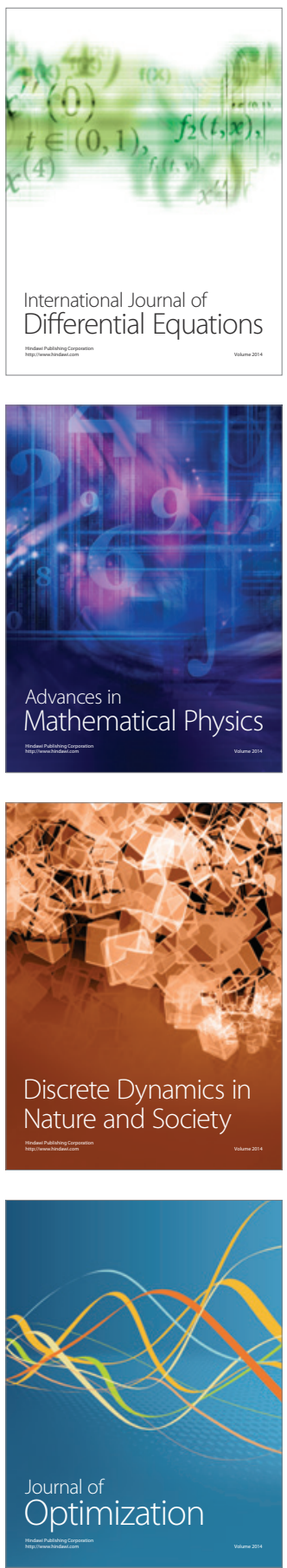